\title{
Influence of cigarette smoking on hormone and lipid metabolism in women in late reproductive
} stage

\author{
Małgorzata Szkup' \\ Anna Jurczak ${ }^{2}$ \\ Beata Karakiewicz ${ }^{3}$ \\ Artur Kotwas ${ }^{3}$ \\ Jacek Kopeć ${ }^{4}$ \\ Elżbieta Grochans' \\ 'Department of Nursing, ${ }^{2}$ Department \\ of Clinical Nursing, ${ }^{3}$ Department of \\ Public Health, Pomeranian Medical \\ University in Szczecin, Szczecin, \\ Poland; ${ }^{4}$ School of Population and \\ Public Health, Faculty of Medicine, \\ The University of British Columbia, \\ Vancouver, BC, Canada
}

Correspondence: Elżbieta Grochans Department of Nursing, Pomeranian Medical University in Szczecin, Żołnierska St 48, 7I-2I 0 Szczecin, Poland

Tel +48914800910

Fax +48 9l 4800905

Email grochans@pum.edu.pl
This article was published in the following Dove Press journal:

Clinical Interventions in Aging

Background: The aim of the study was to analyze lipid and hormone metabolism, body mass index (BMI), and age parameters in late reproductive stage women in relation to cigarette smoking.

Methods: The study enrolled 345 healthy late reproductive stage women living in Poland; $13.33 \%$ were smokers. The first part of the study assessed lipid metabolism (total cholesterol, high-density lipoprotein [HDL], low-density lipoprotein [LDL], and triglycerides) and hormone metabolism (estradiol [E2], follicle-stimulating hormone [FSH], and anti-Müllerian hormone $[\mathrm{AMH}]$ levels) in women in the early phase of the follicular menstrual cycle. The second part of study was carried out using the diagnostic survey method, with a standardized questionnaire (Primary Care Evaluation of Mental Disorders [PRIME-MD]) and the authors' own research tools.

Results: The women were aged $42.3 \pm 4.5$ years (mean \pm SD). The BMI $\left(24.8 \pm 4.04 \mathrm{~kg} / \mathrm{m}^{2}\right)$ did not differ significantly between the groups. The women who smoked cigarettes had a statistically significantly $(p<0.05)$ lower level of HDL as well as higher LDL and triglyceride levels $(p<0.05)$. Differences were also shown in hormone levels: non-smoking participants had statistically significantly higher levels of E2 and FSH $(p<0.05)$. In the group of non-smoking women, age was a predictor exerting a significant positive impact on the levels of total cholesterol, LDL, triglycerides, and AMH $(p<0.05)$. BMI contributed to a decline in HDL and triglyceride levels. In the group of smoking women, age significantly positively influenced the level of E2, and negatively influenced AMH. BMI was associated with a significant decrease in the HDL level.

Conclusion: Smoking cigarettes affects the physical health of women in late reproductive stage through negative influences on lipid and hormone metabolism, among other factors. Age is an unmodifiable factor adversely affecting both lipids and hormones. Higher BMI has a negative influence on lipid metabolism in both groups of women in this study.

Keywords: smoking, cholesterol profile, gonadal steroid hormones

\section{Introduction}

Smoking cigarettes is a well-documented risk factor for the development of cardiovascular disease, atherosclerosis, and type 2 diabetes. Several studies have looked at the relationships between cigarette smoking and triglyceride concentration in blood serum, fasting blood glucose, and high-density lipoprotein (HDL) cholesterol levels. ${ }^{1-5}$ The outcomes did not always demonstrate the expected relationship. The study conducted by Freeman et al on a group of men proved only a slight influence of smoking on the level of triglycerides. ${ }^{2}$ Other reports did not show statistically significant differences 
in fasting glycemia level and insulin resistance between smokers and non-smokers. ${ }^{6,7}$ An important factor influencing these relationships may be body mass, as an increase in body mass is strongly associated with an increase in triglyceride levels in serum ${ }^{8}$ and a decrease in HDL concentration. ${ }^{9}$ Some studies report that people who smoke tend to maintain a lower body weight than non-smokers. ${ }^{10}$

Moreover, epidemiological studies reveal a negative impact of cigarette smoking on ovarian function and fertility, as well as faster onset of menopause. ${ }^{11}$ The effect of environmental factors, such as cigarette smoking and obesity, on ovarian reserve may be important for determining the time of menopause. There is more and more scientific evidence of the usefulness of anti-Müllerian hormone (AMH) testing, together with follicle-stimulating hormone (FSH), in measuring ovarian reserve and evaluating the impact of environmental factors on fertility. ${ }^{12}$

AMH belongs to the transforming growth factor- $\beta$ family. Initiation of AMH expression in granular layer cells of Graafian follicles takes place during folliculogenesis. AMH expression is higher in precavernous granular cells and in small cavernous follicles, and gradually undergoes changes in further stages of follicular development. ${ }^{13}$ Studies conducted among women have mainly focused on analyzing the role of $\mathrm{AMH}$ in serum as a determinant of ovarian function. van Rooij et al found a strong relationship between the number of cavernous follicles, evaluated with the use of ultrasonography, which reflects the size of the primary follicle pool, and the level of AMH in serum. ${ }^{14}$ This suggests that AMH is an excellent marker of ovarian reserve. Studies by Riggs et al revealed that this hormone has strong predictive properties of ovarian reserve, after taking into account age as well as FSH, luteinizing hormone ( $\mathrm{LH})$, and estradiol (E2) hormone levels. ${ }^{15}$

The process of ovarian aging is also characterized by a progressive increase in FSH concentration in serum. This increase is associated with progressive loss of ovarian function. ${ }^{12}$ In murine studies, it was noted that a lack of AMH causes follicles to be recruited at a significantly faster pace. Moreover, it was shown that follicles display an increased sensitivity to $\mathrm{FSH}^{16}$

The 2001 Stages of Reproductive Aging Workshop (STRAW) standardized the classification of menopause status. ${ }^{17}$ A decade later, a new, updated STRAW+10 version was developed. The study presented here included women who, according to this classification, were in the late reproductive stage (the -3 stage), ie, the stage typified by the onset of a decline in fecundability and first changes in the menstrual cycle. In accordance with the STRAW +10 criteria, this phase is divided into two subphases: $-3 b$ and $-3 a$. The length of the menstrual cycle in the $-3 b$ subphase remains unchanged. What can be observed, however, is that the level of AMH and the antral follicle count decrease. The -3 a subphase is characterized by a shortening of the menstrual cycle. Moreover, in the early follicular phase, the level of FSH increases, while the levels of other biomarkers of ovarian aging decrease. ${ }^{17}$ The level of FSH $>30 \mathrm{mIU} / \mathrm{mL}$ is regarded as critical and is marked by a lack of ovarian follicles in the ovaries. In this period, the level of E2 decreases dramatically (from 40-400 pg/mL before menopause, depending on the phase of the menstrual cycle, to $20-30 \mathrm{pg} / \mathrm{mL}$ after menopause). ${ }^{18}$

The aim of this study was to analyze the influence of smoking on:

1) the lipid and hormonal profiles of women in the late reproductive stage;

2) the relationship between hormonal and lipid profiles in the study sample;

3) parameters of hormonal and lipid metabolism depending on the participants' age and body mass index (BMI).

\section{Materials and methods}

The study enrolled healthy women in the late reproductive stage, living in north-west Poland. All participants came from the general population of the West Pomerania Province (Poland). A quota sampling method was applied. Recruitment was performed based on advertisements in public places and local newspapers. All the women were classified into the late reproductive stage group based on the results of their FSH and AMH levels according to STRAW+10 criteria. ${ }^{17,18}$ The youngest participant was 35 years old, and the oldest one was 53 years old. Women with endocrine disorders, thyroid diseases, diabetes, neoplastic disease, abnormal smear test results, or mental disorders were excluded. Women with Axis I mental disorders according to the ICD-10 classification were excluded from the analysis by means of the Primary Care Evaluation of Mental Disorders (PRIME-MD) questionnaire and a psychiatric examination. The study involved 345 women; 26 women were excluded, either because they did not meet the inclusion criteria or because of incomplete data.

All subjects gave their written informed consent for inclusion in the research. The study was conducted in accordance with the Declaration of Helsinki, and the protocol was approved by the Bioethical Commission of the Pomeranian Medical University in Szczecin, Poland (permission number KB-0012/12/12). Those responsible for the whole research 
process were qualified and properly trained academic workers (Masters of Nursing) of the Pomeranian Medical University in Szczecin.

\section{Assessments}

The first part of the study comprised laboratory tests including lipid metabolism (total cholesterol, HDL cholesterol, low-density lipoprotein [LDL] cholesterol, and triglycerides) and hormone metabolism (E2, FSH, and AMH levels).

Women in the early phase of the follicular menstrual cycle had their venous blood sample taken using the closed blood collection system BD Vacutainer ${ }^{\circledR}$, following the existing guidelines. Blood was collected in the treatment room and delivered to the laboratory in accordance with the relevant rules and procedures.

The levels of AMH, FSH, E2, total cholesterol, HDL cholesterol, LDL cholesterol, and triglycerides were measured in a certified laboratory (ISO 9001:2008 certificate).

The following norms of "Laboratoria Medis" were followed: total cholesterol 115-190 mg/dL, HDL cholesterol $\geq 45 \mathrm{mg} / \mathrm{dL}$, LDL cholesterol $<115 \mathrm{mg} / \mathrm{dL}$, and triglycerides $\leq 150 \mathrm{mg} / \mathrm{dL}$. For the follicular phase, the norms were E2 $12.5-166 \mathrm{pg} / \mathrm{mL}$ and FSH $3.5-12.5 \mathrm{mIU} / \mathrm{mL}$.

In the second part of the study, we carried out a survey with a standardized questionnaire (PRIME-MD) and our own research tools, concerning sociodemographic data and information about smoking. Based on the ICD-10 criteria, we established whether the women were addicted to nicotine. The group of smokers comprised only those women who had at least three of the following symptoms during the previous year: a strong need for nicotine; an increasing problem with control of smoking-related behaviors; withdrawal syndrome while making attempts to limit or give up smoking; a growing need to smoke more cigarettes to achieve the same effect that was previously attainable with a lower number; neglecting interests and activities for smoking; and smoking despite the awareness of its numerous negative consequences. ${ }^{19}$ Other women were included in the non-smoking group after confirming that they did not smoke at all.

In addition, the patients were weighed and measured in order to calculate their BMI values according to the formula BMI $=$ weight $(\mathrm{kg}) /$ height $^{2}\left(\mathrm{~m}^{2}\right)$.

\section{Statistical analysis}

Statistical analysis was conducted with the use of the PASW Statistics 18 software. The lack of normal distribution of variables analyzed was confirmed with the Shapiro-Wilk test $(p<0.05)$. The Mann-Whitney $U$-test was applied to verify the null hypothesis regarding the independence of the analyzed variables. Multiple regression analysis was applied to identify predictors having a significant impact on response (or dependent) variables. In the process of statistical inference, statistically significant values were taken as $p \leq 0.05$.

\section{Results}

There were 345 women in the study. Of those, $13.3 \%$ were smokers and $86.7 \%$ were non-smokers. They were aged $42.3 \pm 4.5$ years (mean $\pm \mathrm{SD}$ ), and the median age of smoking and non-smoking women was similar. Three-quarters of the participants $(75.1 \%)$ had a university education and $22.6 \%$ had a secondary education. The majority of the women $(72.5 \%)$ lived in cities with a population greater than 100,000 residents. Most of the participants (75.1\%) lived with partners and the vast majority $(96.5 \%)$ were working.

The BMI of the studied women was $24.8 \pm 4.04 \mathrm{~kg} / \mathrm{m}^{2}$ (mean $\pm \mathrm{SD}$ ), which is within the normal range. The BMI was $24.6 \pm 3.7 \mathrm{~kg} / \mathrm{m}^{2}$ in the non-smoking group and $25.4 \pm 5.4 \mathrm{~kg} / \mathrm{m}^{2}$ in the smoking group (non-significant difference).

The mean values of lipid metabolism indicators for the studied women were within the normal range for triglycerides $(93.16 \pm 50.00 \mathrm{mg} / \mathrm{dL})$ and HDL cholesterol $(55.40 \pm 13.30 \mathrm{mg} / \mathrm{dL})$ and slightly above the existing norms for total cholesterol $(193.05 \pm 33.93 \mathrm{mg} / \mathrm{dL})$ and LDL cholesterol (119.04 $\pm 30.16 \mathrm{mg} / \mathrm{dL})$.

A comparative analysis of lipid metabolism in the smoking and non-smoking women showed statistically significant differences in mean concentrations of HDL, LDL, and triglycerides (Table 1). The women who smoked had lower HDL, and higher LDL and triglyceride levels compared with non-smoking women in the late reproductive stage. Statistically significant differences were also shown in hormone levels, with non-smoking participants having higher levels of E2 (85.9 vs $64.4 \mathrm{pg} / \mathrm{mL}$ ) and FSH (6.2 vs $7.4 \mathrm{mIU} / \mathrm{mL}$ ) compared with smokers (Table 1).

Table 2 presents the results of multiple regression analysis of lipid and hormone levels in both smokers and non-smokers. Estimated models described the actual data in an unsatisfactory way $(R<0.5)$; the differences were not statistically significant $(p>0.05)$.

Next, we analyzed how age and BMI affected lipid and hormone levels in the groups of smokers and non-smokers. In the case of non-smoking women, age was a significant positive contributor to the levels of total cholesterol, LDL, triglycerides, and AMH $(p<0.05)$. BMI was associated with a decrease in HDL and triglyceride levels. In the group of 
Table I Comparative analysis of lipid metabolism and hormones in non-smoking and smoking women in the late reproductive stage

\begin{tabular}{|c|c|c|c|c|c|c|}
\hline \multirow{3}{*}{$\begin{array}{l}\text { Variable } \\
\mathbf{N}=345\end{array}$} & \multicolumn{2}{|l|}{ Non-smoking } & \multicolumn{2}{|l|}{ Smoking } & \multirow[t]{3}{*}{$\mathbf{Z}$} & \multirow[t]{3}{*}{$p$-value } \\
\hline & \multicolumn{2}{|l|}{$n=299(86.67 \%)$} & \multicolumn{2}{|l|}{$n=46(13.33 \%)$} & & \\
\hline & Median (IQR) & Min-Max & Median (IQR) & Min-Max & & \\
\hline \multicolumn{7}{|l|}{ Lipid profile } \\
\hline Total cholesterol (mg/dL) & I9| (44) & $107-295$ & 197.5 (39) & $136-282$ & -1.603 & 0.109 \\
\hline HDL cholesterol (mg/dL) & $55(16)$ & $21-109$ & $5 I(2 I)$ & 18-85 & 1.985 & 0.047 \\
\hline LDL cholesterol (mg/dL) & $116(38)$ & $4 I-22 I$ & $122.5(37)$ & $5 I-203$ & -2.008 & 0.045 \\
\hline Triglycerides (mg/dL) & $79(46)$ & $35-327$ & $97(58)$ & $43-429$ & -2.259 & 0.024 \\
\hline \multicolumn{7}{|l|}{ Hormone profile } \\
\hline E2 (pg/mL) & $85.9(99.5)$ & $4.3-84 I$ & $64.4(46.3)$ & $4.9-279.3$ & 2.333 & 0.020 \\
\hline $\mathrm{FSH}(\mathrm{mlU} / \mathrm{mL})$ & $6.2(3.4)$ & $2.3-497.5$ & $7.4(4.8)$ & $3.8-115.0$ & -2.115 & 0.034 \\
\hline $\mathrm{AMH}(\mathrm{ng} / \mathrm{mL})$ & $\mathrm{I} .33(2.37)$ & $0.15-11.59$ & $1.35(2.14)$ & $0.15-8.17$ & -0.282 & 0.778 \\
\hline
\end{tabular}

Note: Bold values denote statistical significance $(p<0.05)$.

Abbreviations: IQR, interquartile range; Min, minimum value; Max, maximum value; HDL, high-density lipoprotein; LDL, low-density lipoprotein; E2, estradiol; FSH, follicle-stimulating hormone; AMH, anti-Müllerian hormone.

smoking women, age had a significant, positive impact on the level of E2, and a negative impact on AMH. BMI was associated with a significant decline in the HDL level (Table 3).

\section{Discussion}

The outcomes of the research described in this article show adverse effects of smoking cigarettes on lipid metabolism (lower HDL, and higher LDL and triglyceride levels in smokers) and hormonal functioning (lower E2 and higher FSH levels). Factors such as age and a higher BMI make this relationship even stronger.

Dyslipidemia is considered one of the most important modifiable factors in the development of type 2 diabetes, ${ }^{20,21}$ atherosclerosis, ${ }^{22,23}$ cardiovascular disease, ${ }^{24,25}$ and stroke. ${ }^{26,27}$ A well-documented effect of smoking is hypercholesterolemia, which is associated with a higher risk of cardiovascular disease. This factor is believed to influence the increase in total cholesterol level, LDL, and triglycerides, simultaneously inducing the decrease in HDL. ${ }^{28}$ In our study, significant differences between groups of smoking and non-smoking women were shown. Significantly higher concentrations of LDL cholesterol and triglycerides were observed in smoking compared with non-smoking women, while the level of HDL cholesterol was significantly lower in smokers than in non-smokers. These results show that the risk of developing the above-mentioned diseases is considerably higher in smoking women than in their non-smoking counterparts.

A study conducted on 11,559 participants from the Republic of Korea, whose smoking status was verified with a nicotine urine test, revealed that the risk of developing metabolic syndrome was significantly higher among smokers. ${ }^{29}$ The women who smoked had a significantly higher risk of abdominal obesity and a higher level of triglycerides compared with non-smokers. ${ }^{29}$ These findings correspond with our results, showing that smokers significantly more often had symptoms typical of metabolic syndrome, such as lower HDL and higher LDL levels.

The outcomes of a lipid profile assessment performed in women in 1983 suggested a negative influence of smoking on lipid metabolism. ${ }^{30}$ Mean concentrations of triglycerides and total cholesterol in the smoking and non-smoking groups in that study were lower than those obtained in the current study in 2016 (total cholesterol - 1983: smoking

Table 2 Multiple regression analysis of lipid metabolism and hormones in smoking and non-smoking women in the late reproductive stage

\begin{tabular}{lllllll}
\hline & Smoking & $\begin{array}{l}\text { Total cholesterol } \\
(\mathbf{m g} / \mathbf{d L})\end{array}$ & $\begin{array}{l}\text { HDL cholesterol } \\
(\mathbf{m g} / \mathbf{d L})\end{array}$ & $\begin{array}{l}\text { LDL cholesterol } \\
(\mathbf{m g} / \mathbf{d L})\end{array}$ & $\begin{array}{l}\text { Triglycerides } \\
(\mathbf{m g} / \mathbf{d L})\end{array}$ & $R / \mathbf{p}$ \\
\hline AMH $(\mathrm{ng} / \mathrm{mL})$ & Yes & - & -0.015 & -0.002 & 0.001 & $0.324 / 0.312$ \\
& No & 0.000 & 0.003 & - & -0.001 & $0.120 / 0.375$ \\
E2 $(\mathrm{pg} / \mathrm{mL})$ & Yes & - & 0.082 & 0.232 & -0.036 & $0.270 / 0.516$ \\
& No & 0.112 & 0.583 & - & 0.172 & $0.115 / 0.417$ \\
FSH $(\mathrm{mlU} / \mathrm{mL})$ & Yes & - & -0.068 & 0.097 & -0.039 & $0.300 / 0.400$ \\
& No & 0.004 & 0.000 & - & -0.026 & $0.073 / 0.810$ \\
\hline
\end{tabular}

Abbreviations: $\mathrm{HDL}$, high-density lipoprotein; LDL, low-density lipoprotein; $\mathrm{AMH}$, anti-Müllerian hormone; E2, estradiol; FSH, follicle-stimulating hormone; $R$, coefficient of determination; $p$, level of significance. 
Table 3 Multiple regression analysis of hormone and lipid metabolism determinants with age and BMI in the groups of smoking and non-smoking women in the late reproductive stage

\begin{tabular}{lllll}
\hline & Smoking & Age & BMI & $R / p$ \\
\hline Total cholesterol & Yes & $0.247^{*}$ & $-0.247^{*}$ & $0.340 / 0.067$ \\
(mg/dL) & No & $0.154^{* *}$ & 0.030 & $0.161 / 0.021$ \\
HDL cholesterol & Yes & 0.193 & $-0.382^{* *}$ & $0.418 / 0.014$ \\
(mg/dL) & No & -0.066 & $-0.4 I I^{* * *}$ & $0.425 / 0.000$ \\
LDL cholesterol & Yes & 0.197 & -0.157 & $0.246 / 0.255$ \\
(mg/dL) & No & $0.169^{* *}$ & $0.095^{*}$ & $0.205 / 0.002$ \\
Triglycerides & Yes & -0.018 & 0.895 & $0.134 / 0.671$ \\
(mg/dL) & No & $0.108^{* *}$ & $0.390^{* * *}$ & $0.418 / 0.000$ \\
AMH $(\mathrm{ng} / \mathrm{mL})$ & Yes & $-0.402^{* *}$ & 0.095 & $0.408 / 0.018$ \\
& No & $-0.347^{* * *}$ & -0.063 & $0.361 / 0.000$ \\
E2 (pg/mL) & Yes & $0.37 I^{* *}$ & $0.233^{*}$ & $0.448 / 0.007$ \\
& No & 0.042 & -0.076 & $0.082 / 0.369$ \\
FSH $(\mathrm{mlU} / \mathrm{mL})$ & Yes & $0.243^{*}$ & -0.231 & $0.327 / 0.083$ \\
& No & 0.069 & -0.078 & $0.097 / 0.245$ \\
\hline
\end{tabular}

Notes: $* p<0.1 ; * * p<0.05 ; * * * p<0.001$.

Abbreviations: BMI, body mass index; HDL, high-density lipoprotein; LDL, lowdensity lipoprotein; AMH, anti-Müllerian hormone; E2, estradiol; FSH, folliclestimulating hormone; $R$, coefficient of determination; $p$, level of significance.

$189 \mathrm{mg} / \mathrm{dL}$ and non-smoking $197 \mathrm{mg} / \mathrm{dL}$ vs 2016: $201 \mathrm{mg} / \mathrm{dL}$ and $191 \mathrm{mg} / \mathrm{dL}$, respectively; triglycerides - 1983: smoking $100 \mathrm{mg} / \mathrm{dL}$ and non-smoking $64 \mathrm{mg} / \mathrm{dL}$ vs 2016: $114 \mathrm{mg} / \mathrm{dL}$ and $89 \mathrm{mg} / \mathrm{dL}$, respectively). ${ }^{30}$ These differences may be caused by the influence of environmental factors on lipid metabolism.

Studies conducted in the Tobacco Cessation Clinic indicate that quitting smoking, tested in the third week from cessation, resulted in increased body weight. ${ }^{31}$ Despite this effect, it was noted that the risk of metabolic syndrome decreased owing to a reduction in the number of people with hypertension, hypertriglyceridemia, and low HDL levels. The benefits from smoking cessation outweighed the adverse effects of weight gain. ${ }^{31}$ Conclusions from this study suggest that irrespective of its early consequences (such as an increase in body mass), giving up smoking should definitely be encouraged to reduce the risk of developing diseases of civilization.

In a study by Qi et al, the risk of developing dyslipidemia in women increased with age, reaching its peak value at 60 years of age. ${ }^{32}$ This relationship is believed to result from hormonal changes which occur in the premenopausal and postmenopausal periods..$^{32}$ The age of women in the smoking and non-smoking groups in that study was very similar. Our analysis has shown that age plays a major role in lipid metabolism in women, both in smokers and in non-smokers.

The study carried out by Ruan and Mueck revealed that duration and intensity of smoking considerably affect both endogenous and exogenous E2 levels and may cause a decrease or inactivation of this hormone. ${ }^{33}$ It was noted that cigarette smoking reverses the beneficial effects of estrogens in reducing hot flashes and genitourinary symptoms. Furthermore, the positive influence of estrogens on lipid metabolism and the risk of cardiovascular system diseases is reduced. ${ }^{33}$ In this study, the level of E2 was significantly lower in the group of smoking women than in non-smokers (median $64.4 \mathrm{pg} / \mathrm{mL}$ vs $85.9 \mathrm{pg} / \mathrm{mL}$ ), which corresponds with the results reported by Ruan and Mueck, even though we did not take into account the intensity of smoking, only the fact of being addicted to nicotine.

Research conducted among 141 infertile women in Chile proved that cigarette smoking is a risk factor leading to decreased AMH concentrations in blood plasma. ${ }^{34}$ After ruling out the influence of other factors, it was shown that smoking caused a decrease of AMH by $2.29 \mathrm{ng} / \mathrm{mL}$, irrespective of the number of cigarettes smoked in the week preceding the test. However, a relationship between smoking and FSH and estradiol levels on the third day of the menstrual cycle was not proven. ${ }^{34}$ In contrast, the level of AMH in our study was similar in both groups, but a significant relationship between smoker status and estradiol and FSH levels in the initial follicular phase was demonstrated. Divergence between these results was probably associated with the fact that the Chilean study included infertile women with hormonal disorders, whereas the present study involved healthy women in the late reproductive stage (FSH: smokers $14.86 \mathrm{mIU} / \mathrm{mL}$ vs $7.4 \mathrm{mIU} / \mathrm{mL}$ and non-smokers $14.03 \mathrm{mIU} / \mathrm{mL}$ vs $6.2 \mathrm{mIU} / \mathrm{mL}$, in the Chilean and the present study, respectively; E2: smokers $28.89 \mathrm{pg} / \mathrm{mL}$ vs $64.4 \mathrm{pg} / \mathrm{mL}$ and non-smokers $30.67 \mathrm{pg} / \mathrm{mL}$ vs $85.9 \mathrm{pg} / \mathrm{mL}$ ). Comparison between these two studies reveals the influence of smoking on female hormone metabolism, depending on whether hormonal function was normal or disordered.

A study among Chinese women showed that BMI has a significant impact on the occurrence of dyslipidemia. ${ }^{32}$ It was observed that people with dyslipidemia had a BMI of $25.1 \pm 3.3 \mathrm{~kg} / \mathrm{m}^{2}$ (mean $\pm \mathrm{SD}$ ), while for people with no lipid metabolism abnormalities, the BMI was $23.2 \pm 3.1$ $\mathrm{kg} / \mathrm{m}^{2}$. This relationship was particularly apparent for people with hypertriglyceridemia, in whom the BMI was $25.5 \pm 3.4 \mathrm{~kg} / \mathrm{m}^{2}$. Moreover, results of multivariable logistic regression revealed that the level of education, presence of obesity, and cigarette smoking significantly increased the risk of dyslipidemia, while regular physical activity turned out to be an independent protective factor. ${ }^{32}$ Our study also demonstrated a significant negative impact of increased BMI 
on lipid metabolism, which especially refers to the levels of HDL and triglycerides. This effect was observed in both smoking and non-smoking women.

In the study by Qi et al, the age-adjusted prevalence of dyslipidemia was higher in women $(37.6 \%)$ than in men $(34.4 \%){ }^{32}$ This result may be due to differences in the frequency of overweight, obesity, and central obesity, which affect women much more often than men in the Chinese population. ${ }^{31}$ In the current study, the level of cholesterol in smoking and non-smoking women in the late reproductive stage increased significantly with age. Confirmation of these findings requires further analyses and a larger number of studied patients.

\section{Limitations}

Our study was not free from limitations. The most important of these were the small number of women categorized as smokers, and the fact that women were included in the non-smoking group exclusively on the basis of the ICD-10 dependence syndrome. It would be interesting to conduct more extensive research, taking into consideration duration and intensity of smoking, as well as exposure to passive smoking. Despite these limitations, research outcomes indicate the presence of mutual relationships between some hormone and lipid metabolism markers and cigarette smoking in the group of women in the late reproductive stage.

\section{Conclusion}

In relation to our earlier aims we conclude that 1) cigarette smoking in women in the late reproductive stage has a negative influence on lipid and hormone metabolism; 2) age affects both lipid and hormone metabolism in women in the late reproductive stage. This relationship can be more clearly seen in non-smoking women; and 3) higher BMI values have a negative influence on lipid metabolism in both smoking and non-smoking women.

\section{Disclosure}

The authors declare no conflicts of interest in this work.

\section{References}

1. Håglin LM, Törnkvist B, Bäckman LO. High serum phosphate and triglyceride levels in smoking women and men with CVD risk and type 2 diabetes. Diabetol Metab Syndr. 2014;6(1):39.

2. Freeman DJ, Griffin BA, Murray E, et al. Smoking and plasma lipoproteins in man: effects on low density lipoprotein cholesterol levels and high density lipoprotein subfraction distribution. Eur J Clin Invest. 1993;23(10):630-640.

3. Kuzuya M, Ando F, Iguchi A, Shimokata H. Effect of smoking habit on age-related changes in serum lipids: a cross-sectional and longitudinal analysis in a large Japanese cohort. Atherosclerosis. 2006;185(1):183-190.
4. Reaven G, Tsao PS. Insulin resistance and compensatory hyperinsulinemia: the key player between cigarette smoking and cardiovascular disease? J Am Coll Cardiol. 2003;41(6):1044-1047.

5. Houston TK, Person SD, Pletcher MJ, Liu K, Iribarren C, Kiefe CI. Active and passive smoking and development of glucose intolerance among young adults in a prospective cohort: CARDIA study. $B M J$. 2006;332(7549):1064-1069.

6. Soulimane S, Simon D, Herman WH, et al. $\mathrm{HbA}_{1 \mathrm{c}}$, fasting and $2 \mathrm{~h}$ plasma glucose in current, ex- and never-smokers: a meta-analysis. Diabetologia. 2014;57(1):30-39.

7. Masulli M, Riccardi G, Galasso R, Vaccaro O. Relationship between smoking habits and the features of the metabolic syndrome in a nondiabetic population. Nutr Metab Cardiovasc Dis. 2006;16(5):364-370.

8. Taniguchi A, Nakai Y, Sakai M, et al. Relationship of regional adiposity to insulin resistance and serum triglyceride levels in nonobese Japanese type 2 diabetic patients. Metabolism. 2002;51(5):544-548.

9. Ross R, Aru J, Freeman J, Hudson R, Janssen I. Abdominal adiposity and insulin resistance in obese men. Am J Physiol Endocrinol Metab. 2002;282(3):E657-E663.

10. Akbartabartoori M, Lean ME, Hankey CR. Relationships between cigarette smoking, body size and body shape. Int J Obes (Lond). 2005;29(2): 236-243.

11. Sowers MF, La Pietra M. Menopause: its epidemiology and potential association with chronic diseases. Epidemiol Rev. 1995;17:287-302.

12. Sowers MR, McConnell D, Yosef M, Jannausch ML, Harlow SD, Randolph JF Jr. Relating smoking, obesity, insulin resistance and ovarian biomarker changes to the final menstrual period. Ann N Y Acad Sci. 2010;1204:95-103.

13. Durlinger ALL, Visser JA, Themmen APN. Regulation of ovarian function: the role of anti-Müllerian hormone. Reproduction. 2002;124: 601-609.

14. van Rooij IAJ, Broekmans FJM, te Velde ER, et al. Serum antiMüllerian hormone levels: a novel measure of ovarian reserve. Hum Reprod. 2002;17:3065-3071.

15. Riggs RM, Duran EH, Baker MW, Kimble TD, Hobeika E, Yin L, et al. Assessment of ovarian reserve the anti-mullerian hormone: a comparison of the predictive value of anti-mullerian hormone, follicle stimulating hormone, inhibin B and age. Am J Obstet Gynecol. 2008;199:202.

16. Durlinger AL, Gruijters MJ, Kramer P, et al. Anti-Müllerian hormone attenuates the effects of FSH on follicle development in the mouse ovary. Endocrinology. 2001;142:4891-4899.

17. Harlow SD, Gass M, Hall JE, et al. Executive summary of the Stages of Reproductive Aging Workshop + 10: addressing the unfinished agenda of staging reproductive aging. Menopause. 2012;19(4):387-395.

18. Baranowski W. Przemiany hormonalne wieku menopauzalnego [Hormonal changes of menopausal age]. In: Pertyński T, editor. Diagnostyka i terapia wieku menopauzalnego [Diagnosis and therapy of menopausal age]. Wrocław: Urban \& Partner; 2004:1-10. Polish.

19. DiFranza J, Ursprung WW. A systematic review of the International Classification of Diseases criteria for the diagnosis of tobacco dependence. Addict Behav. 2010;35(9):805-810.

20. Jayarama N, Lakshmaiah MR. Prevalence and pattern of dyslipidemia in type 2 diabetes mellitus patients in a rural tertiary care centre, southern India. Glob J Med Public Health. 2012;1:24-27.

21. Zhou X, Zhang W, Liu X, Li Y. Interrelationship between diabetes and periodontitis: role of hyperlipidemia. Arch Oral Biol. 2015;60: 667-674.

22. Snehalatha C, Nanditha A, Shetty AS, Ramachandran A. Hypertriglyceridaemia either in isolation or in combination with abdominal obesity is strongly associated with atherogenic dyslipidaemia in Asian Indians. Diabetes Res Clin Pract. 2011;94:140-145.

23. Berenson GS, Srinivasan SR, Bao W, Newman WP, Tracy RE, Wattigney WA. Association between multiple cardiovascular risk factors and atherosclerosis in children and young adults. The Bogalusa Heart Study. N Engl J Med. 1998;338:1650-1656. 
24. Stamler J, Daviglus ML, Garside DB, Dyer AR, Greenland P, Neaton JD. Relationship of baseline serum cholesterol levels in 3 large cohorts of younger men to long-term coronary, cardiovascular, and all-cause mortality and to longevity. JAMA. 2000;284:311-318.

25. Vergani C, Lucchi T. Plasma HDL cholesterol and risk of myocardial infarction. Lancet. 2012;380:1989-1990.

26. Djelilovic-Vranic J, Alajbegovic A, Zelija-Asimi V, et al. Predilection role diabetes mellitus and dyslipidemia in the onset of ischemic stroke. Med Arch. 2013;67:120-123.

27. Tziomalos K, Athyros VG, Karagiannis A, Mikhailidis DP. Dyslipidemia as a risk factor for ischemic stroke. Curr Top Med Chem. 2009;9: 1291-1297.

28. Chelland Campbell S, Moffatt RJ, Stamford BA. Smoking and smoking cessation - the relationship between cardiovascular disease and lipoprotein metabolism: a review. Atherosclerosis. 2008;201(2):225-235.

29. Kang JH, Song YM. Association between cotinine-verified smoking status and metabolic syndrome: analyses of Korean National Health and Nutrition Examination Surveys 2008-2010. Metab Syndr Relat Disord. 2015;13(3):140-148.
30. Willett W, Hennekens CH, Castelli W, et al. Effects of cigarette smoking on fasting triglyceride, total cholesterol, and HDL-cholesterol in women. Am Heart J. 1983;105(3):417-421.

31. Ponciano-Rodriguez G, Paez-Martinez N, Villa-Romero A, GutierrezGrobe Y, Mendez-Sanchez N. Early changes in the components of the metabolic syndrome in a group of smokers after tobacco cessation. Metab Syndr Relat Disord. 2014;12(4):242-250.

32. Qi L, Ding X, Tang W, Li Q, Mao D, Wang Y. Prevalence and risk factors associated with dyslipidemia in Chongqing, China. Int J Environ Res Public Health. 2015;12:13455-13465.

33. Ruan X, Mueck AO. Impact of smoking on estrogenic efficacy. Climacteric. 2015;18(1):38-46.

34. Fuentes A, Escalona J, Céspedes P, Repetto V, Iñiguez G. El hábito de fumar se asocia a baja concentración plasmática de hormona antimülleriana en mujeres infértiles [Effects of smoking on plasma antimüllerian hormone concentrations among infertile women]. Rev Med Chil. 2013;141(1):23-27.
Clinical Interventions in Aging

\section{Publish your work in this journal}

Clinical Interventions in Aging is an international, peer-reviewed journal focusing on evidence-based reports on the value or lack thereof of treatments intended to prevent or delay the onset of maladaptive correlates of aging in human beings. This journal is indexed on PubMed Central, MedLine,

\section{Dovepress}

CAS, Scopus and the Elsevier Bibliographic databases. The manuscript management system is completely online and includes a very quick and fair peer-review system, which is all easy to use. Visit http://www.dovepress. $\mathrm{com} /$ testimonials.php to read real quotes from published authors. 